\title{
Reflection of Electromagnetic Waves From a Lossy Magnetoplasma
}

\author{
James R. Wait and Lillie C. Walters
}

\author{
Contribution From the Central Radio Propagation Laboratory, National Bureau of Standards, \\ Boulder, Colo.
}

\begin{abstract}
A method is outlined for calculating the reflection coefficient from a horizontally stratified ionized medium. The profiles of electron density and the collision frequencies are both taken to be exponential functions. The d-c magnetic field is taken to be horizontal and transverse to the direction of propagation. The specific results described are applicable to the oblique reflection of VLF radio waves in the $D$ layer of the ionosphere for propagation along the magnetic equator. It is confirmed that the reflection coefficient is nonreciprocal in both amplitude and phase. For a wide range of the parameters, the magnitude of the reflection coefficient is greater for west-to-east propagation than for east-to-west propagation.
\end{abstract}

\section{Introduction}

The lower ionosphere is primarily responsible for the propagation of VLF radio waves to great distances. In theoretical treatments of this problem it is often assumed that the lower edge of the ionosphere may be represented by a sharply bounded and homogeneous ionized medium. Actually, such a model was used by G. N. Watson [1919] over 40 years ago. Applications and refinements of such a model have been discussed frequently in the recent literature [e.g., Budden, 1962; Wait, 1962; Johler, 1963]. One such refinement is the inclusion of the earth's magnetic field in the analysis. This, of course, renders the medium anisotropic. If the vertical inhomogeneity (or horizontal stratification) of the ionosphere is also considered simultaneously, the situation becomes very complicated indeed. An extensive study of analytical methods to treat such problems has been carried out by K. G. Budden and his colleagues at Cambridge University. Much of this work is summarized in a monumental text [Budden, 1961] which will be the standard work on the subject for some time. Budden makes extensive use of "full wave" methods which may be described as a frontal assault on the differential equations satisfied by the field components in the medium.

In this paper we shall consider a special case of a horizontally stratified and anisotropic ionosphere. Specifically, the earth's magnetic field is assumed to be purely transverse to the direction of propagation. Strictly speaking, this is applicable only to the situation when the path of propagation is along the magnetic equator. However, the characteristics in this special case prevail at other latitudes if the transverse component of the field is appreciable. At least this is borne out by a numerical study of the sharply bounded ionosphere for an arbitrary magnetic dip angle [Johler, 1961]. In any case, the resulting simplicity of the differential equations for the limiting case of a purely transverse magnetic field encourages one to consider this situation in more detail. In particular, it is desirable to investigate the influence of gradient of both the electron density and collision frequency. In much of the previous work on this subject the collision frequency has been assumed constant.

In a study of the recent literature [e.g., Belrose, 1963 ] it is found that both the electron density $N(z)$ and the collision frequency $\nu(z)$ vary approximately in an exponential manner with height $z$. For example, in the undisturbed daytime ionosphere we may assume that

$$
N(z)=N_{0} \exp (b z)
$$

and

$$
\nu(z)=\nu_{0} \exp (-a z),
$$

where $a$ and $b$ are positive constants and $z$ is some specified level in the ionosphere. From a study of the experimental data [Belrose, 1963], it appears that, if the reference level is $70 \mathrm{~km}$ above the earth's surface, $N_{0} \sim 10^{2}$ electrons $/ \mathrm{cm}^{3}$ and $\nu_{0} \sim 10^{7} \mathrm{sec}^{-1}$. The gradient parameters are then expected to be given approximately by $b \cong 0.15 \mathrm{~km}^{-1}( \pm 0.1)$ and $a \sim 0.15$ $\mathrm{km}^{-1}( \pm 0.02)$. The quoted values of these constants must be considered tentative and certainly subject to change. Furthermore, it must be understood that significant departures from the exponential shape are to be expected under disturbed conditions.

\section{Formulation}

The situation is shown explicitly in figure 1a. A vertically polarized plane wave is incident at angle $\theta$ on to a horizontally stratified ionosphere. The $z$ axis is taken to be positive in the upward direction. At the reference level $z=0$, the electron density and the collision frequency have values designated by $N_{0}$ and $\nu_{0}$, respectively. The "scale height" which is 


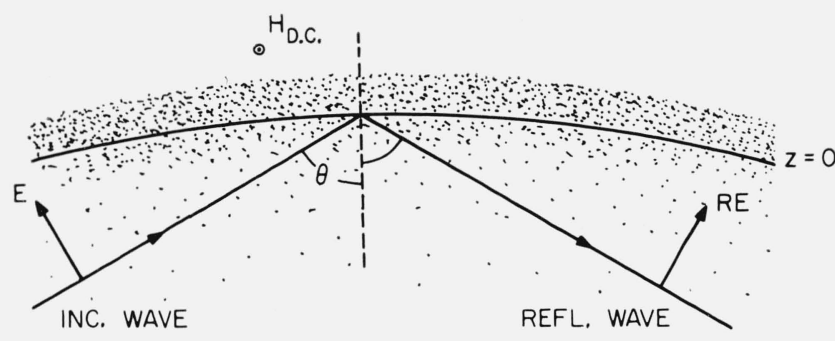

Figure 1a. Illustrating oblique reflection of VLF radio waves from an inhomogeneous (horizontally stratified) ionosphere with a transverse $d-c$ magnetic field.

equal to $1 / \mathrm{b}$ or $1 / \mathrm{a}$, as indicated in figure $1 \mathrm{~b}$ is of the order of $6 \mathrm{~km}$ for both of these profiles. As mentioned above, these are typical of the daytime $D$ layer for both the $N$ and $\nu$ profiles.

The lower ionosphere, which is idealized here as a stratified ionized medium, may be regarded as an electron plasma. The (angular) electron plasma frequency $\omega_{0}$ is thus given by

$$
\omega_{0}^{2}=3.18 \times 10^{9} \times N,
$$

where $N$ is the electron density in electrons per cubic centimeter and $\omega_{0}$ has dimensions of radians per second. The dielectric properties of such a (cold) plasma may be described in terms of a tensor dielectric constant $(\epsilon)$. Thus, the displacement vector $\vec{D}$ and the electric field $\vec{E}$ are connected by

$$
\vec{D}=(\epsilon) \vec{E}
$$

Choosing the d-c magnetic field to be along the axial direction, ${ }^{1}$ the tensor has the form

$$
(\epsilon)=\left(\begin{array}{ccc}
\epsilon^{\prime} & -i q & 0 \\
i q & \epsilon^{\prime} & 0 \\
0 & 0 & \epsilon^{\prime \prime}
\end{array}\right)
$$

where harmonic time dependence according to exp $(+i \omega t)$ is assumed. The coefficients $\epsilon^{\prime}, \epsilon^{\prime \prime}$, and $q$ are given by [Wait, 1962]

$$
\begin{gathered}
\frac{\epsilon^{\prime}}{\epsilon_{0}}=1-\frac{i(\nu+i \omega) \omega_{0}^{2} / \omega}{\omega_{T}^{2}+(\nu+i \omega)^{2}}, \\
\frac{q}{\epsilon_{0}}=-\frac{\omega_{T} \omega_{0}^{2} / \omega}{\omega_{T}^{2}+(\nu+i \omega)^{2}}, \\
\frac{\epsilon^{\prime \prime}}{\epsilon_{0}}=1-\frac{i \omega_{0}^{2}}{(\nu+i \omega) \omega},
\end{gathered}
$$

${ }^{1}$ In what follows, the $x$ axis is taken to be in the axial direction. Thus eq (4) is given by

$$
\left(\begin{array}{l}
D_{y} \\
D_{z} \\
D_{x}
\end{array}\right)=(\epsilon)\left(\begin{array}{l}
E_{y} \\
E_{z} \\
E_{x}
\end{array}\right) \text {. }
$$

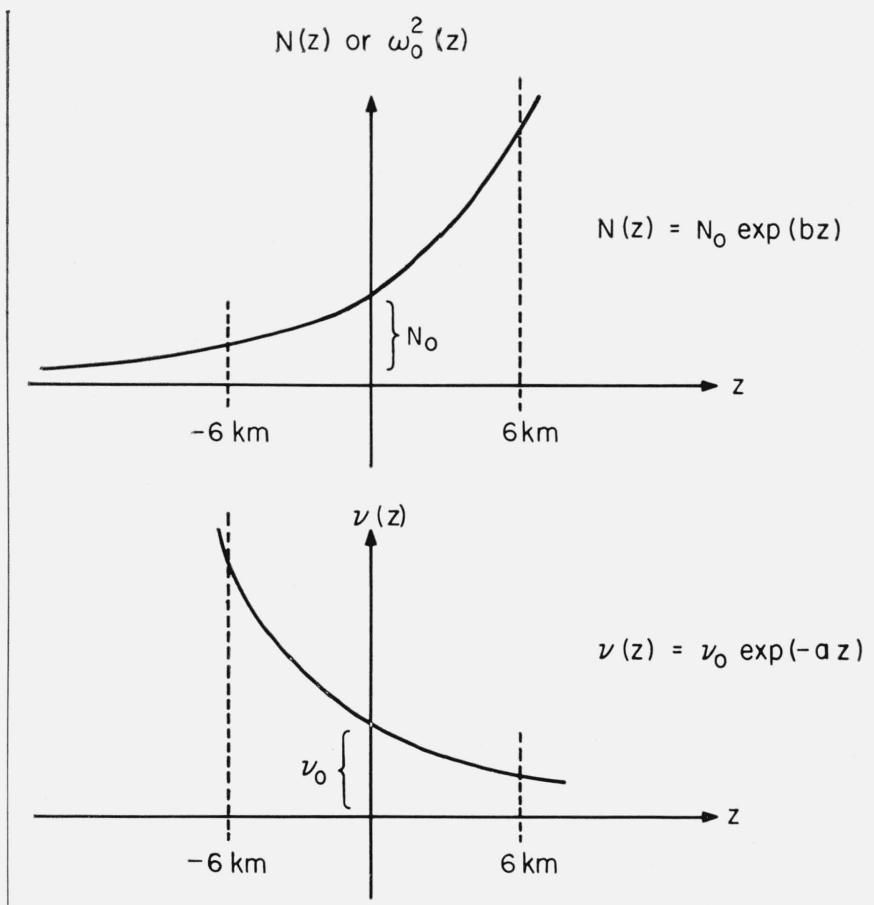

FiguRE 1b. Sketch of the profiles of electron density $\mathrm{N}(\mathrm{z})$ and the collision frequency $\nu(\mathrm{z})$.

where $\epsilon_{0}=8.85 \times 10^{-12}$ is the dielectric constant of free space and $\omega_{T}$ is the (angular) gyro frequency.

Maxwell's equations, which are applicable to this situation, are

$$
\begin{aligned}
& i(\epsilon) \omega \vec{E}=\operatorname{curl} \vec{H}, \\
& -i \mu \omega \vec{H}=\operatorname{curl} \vec{E},
\end{aligned}
$$

where $\mu$ is the magnetic permeability of the plasma. The first equation above may be written

$$
i \omega \vec{E}=\left(\epsilon^{-1}\right) \operatorname{curl} \vec{H}
$$

where

$$
\epsilon_{0}\left(\epsilon^{-1}\right)=\left(\begin{array}{ccc}
M & -i K & 0 \\
i K & M & 0 \\
0 & 0 & \epsilon_{0} / \epsilon^{\prime \prime}
\end{array}\right)
$$

with

$$
M=\frac{\epsilon^{\prime} \epsilon_{0}}{\left(\epsilon^{\prime}\right)^{2}-q^{2}},
$$

and

$$
K=\frac{-q \epsilon_{0}}{\left(\epsilon^{\prime}\right)^{2}-q^{2}} .
$$

For convenience in what follows, we now choose the gyro axis to be in the $x$ direction (i.e., the d-c magnetic field is taken to be parallel to the $x$ direc- 
tion). As a result $\partial / \partial x=0$ since the incident wave is specified to be transverse to the d-c magnetic field. It is now a straightforward matter to show that

$$
\begin{aligned}
& \left(\frac{\partial^{2}}{\partial y^{2}}+\frac{\partial^{2}}{\partial z^{2}}+\frac{k^{2}}{M \hat{M}}\right) H_{x}=0, \\
& \left(\frac{\partial^{2}}{\partial y^{2}}+\frac{\partial^{2}}{\partial z^{2}}+\frac{k^{2} \epsilon^{\prime \prime}}{\hat{M} \epsilon_{0}}\right) E_{x}=0,
\end{aligned}
$$

where $k=\left(\epsilon_{0} \mu_{0}\right)^{\frac{1}{2}} \omega=2 \pi / \lambda$ and $M=\mu_{0} / \mu$. Here $\lambda$ is the free space wavelength and $\mu_{0}$ is the magnetic permeability of free space.

From Maxwell's equations, the other field components may be obtained from $H_{x}$ and $E_{x}$. For example,

$$
\begin{gathered}
i \epsilon_{0} \omega E_{y}=M \frac{\partial H_{x}}{\partial z}+i K \frac{\partial H_{x}}{\partial y}, \\
i \epsilon_{0} \omega E_{z}=i K \frac{\partial H_{x}}{\partial z}-M \frac{\partial H_{x}}{\partial y}, \\
-i \mu_{0} \omega H_{y}=\hat{M} \frac{\partial E_{x}}{\partial z}, \\
-i \mu_{0} \omega H_{z}=-\hat{M} \frac{\partial E_{x}}{\partial y} .
\end{gathered}
$$

It is immediately evident that the general problem splits into two parts. The total field may be regarded as the superposition of two partial fields; one is characterized by $\vec{E}=\left(E_{x}, 0,0\right)$ and the other by $\vec{H}=\left(H_{x}, 0,0\right)$. These may be called TE (transverse electric) and TM (transverse magnetic) waves, respectively. For a vertically polarized incident wave, the first partial field (i.e., the TE waves) is not excited, so further attention is restricted to the TM waves. ${ }^{2}$

\section{Preliminary Problem}

As a simple preliminary problem we shall consider the ionosphere to be sharply bounded and homogeneous. Thus, for $z>0$, the dielectric properties are to be characterized by the tensor $(\epsilon)$ which does not vary with $z$. The region $z<0$ is free space.

The incident wave is of the form

$$
H_{x}^{\mathrm{inc}}=h_{0} e^{-i k C z} e^{-i k S y},
$$

where $h_{0}$ is a constant and where $C=\left(1-S^{2}\right)^{1 / 2}=\cos \theta$ in terms of the angle of incidence $\theta$. Then the reflected wave must be of the form

$$
H_{x}^{\text {refl }}=h_{0} e^{+i k C z} e^{-i k S y} R,
$$

where $R$, by definition, is the reflection coefficient.

${ }^{2}$ Actually, the TE waves in this case are not influenced by the d-c magnetic field and the medium is effectively isotropic.
For the region $z>0$,

$$
H_{x}=f(z) \exp (-i k S y)
$$

where, by virtue of (15), $f(z)$ satisfies

$$
\left[\frac{\partial^{2}}{\partial z^{2}}+k^{2}\left(\frac{1}{M \hat{M}}-S^{2}\right)\right] f(z)=0 .
$$

Therefore,

$$
H_{x}=h_{0} T \exp \left[-i k\left[\frac{1}{M \hat{M}}-S^{2}\right]^{1 / 2} z\right] \exp (-i k S y),
$$

where $T$, by definition, is a transmission coefficient.

The unknown functions $R$ and $T$ are found by applying the boundary conditions which require the continuity of $E_{y}$ and $H_{x}$ at $z=0$. This readily leads to

and

$$
T=\frac{2 C}{C+\Delta},
$$

where

$$
R=\frac{C-\Delta}{C+\Delta},
$$

$$
\Delta=M\left[\frac{1}{M \hat{M}}-S^{2}\right]^{1 / 2}+i K S
$$

If $\hat{M}=\frac{\mu_{0}}{\mu}=1$, which is the usual case,

$$
\Delta=\frac{\left[C^{2}+\frac{1+i L}{i L-L^{2}-\gamma^{2}}\right]^{1 / 2}\left(i L-L^{2}-\gamma^{2}\right)-i \gamma S}{(1+i L)^{2}-\gamma^{2}},
$$

where

$$
L=\frac{(\nu+i \omega) \omega}{\omega_{0}^{2}} \text { and } \gamma=\frac{\omega_{T} \omega}{\omega_{0}^{2}} .
$$

The latter result was given by Barber and Crombie [1959] who employed a somewhat more involved derivation.

\section{The General Problem}

We shall now return to our originally stated problem. The continuous profiles of $N(z)$ and $\nu(z)$ are replaced by a very large, but finite, number of steps. In other words, the inhomogeneous medium is replaced by a stack of thin homogeneous layers. For purposes of discussion, there shall be $P$ such layers while a typical layer is the $p$ th layer. Thus, $p$ ranges from 1 to $P$ through integral values. Somewhere at a sufficiently negative value of $z$, the medium may be regarded as free space. This level is denoted $z=-z_{0}$.

Within the $p$ th layer, the fields may be regarded as a superposition of upgoing and downgoing waves. These are characterized by the functions exp 
$\left(-i \beta_{p} k z\right)$ and $\exp \left(+i \beta_{p} k z\right)$, respectively, where

$$
\beta_{p}=\left[\frac{1}{M_{p} \hat{M}_{p}}-S^{2}\right]^{1 / 2} .
$$

The characteristic impedances associated with these wave types are

$$
K_{p}^{+}=-\frac{E_{y}^{+}}{H_{x}^{+}}
$$

and

$$
K_{p}^{-}=\frac{E_{y}^{-}}{H_{x}^{-}}
$$

where the + sign denotes upgoing and the $-\operatorname{sign}$ denotes downgoing waves. From (17) and (18), it is seen that

and

$$
K_{p}^{+}=\eta_{0}\left(M_{p} \beta_{p}+i K_{p} S\right),
$$

where

$$
K_{p}^{-}=\eta_{0}\left(M_{p} \beta_{p}-i K_{p} S\right)
$$

$$
\eta_{0}=\left(\mu_{0} / \epsilon_{0}\right)^{1 / 2}=120 \pi \text { ohms. }
$$

The problem may now be solved by an application of nonuniform transmission line theory [Schelkunoff, 1943; Wait, 1962]. Thus, the reflection coefficient which is referred to the lower edge of the bottom slab is given by

$$
R_{0}=\frac{K_{0}^{+}-Z_{1}}{K_{0}^{-}+Z_{1}}=\frac{C-\Delta}{C+\Delta},
$$

where $\Delta=Z_{1} / \eta_{0}$ and where $Z_{1}$ is the input impedance at the bottom of layer number 1 . Now, $Z_{1}$ may be expressed in terms of $Z_{2}$ which, in turn, may be expressed in terms of $Z_{3}$. The process is continued until the topmost layer is reached where $Z_{p}=K_{p}^{+}$is assumed known. The details of this derivation are given elsewhere [Wait, 1962].

The required number of layers is best determined by studying the stability of the solution as the number is increased. Because of the relatively long wavelength involved and because of the finite losses in the medium, the solution converges nicely as the number of layers is increased. For the cases discussed here, the step size was never greater than 0.1 $\mathrm{km}$ and this appeared to be smaller than necessary.

\section{Presentation of Results}

The final results of the numerical calculations are presented in such a fashion that the phase of the reflection coefficient $R$ is referred to the level $z=0$. Thus, by definition,

$$
R=\left[R_{0} \exp \left(i 2 k C z_{0}\right)\right]_{z_{0} \rightarrow \infty} .
$$

Physically, this means that the observer at $z=-z_{0}$ is sufficiently far below the ionosphere that the medium may be regarded as free space. In practice $z_{0}$ is chosen to be large enough that the phase of $R$ does not vary with further changes in $z_{0}$. For the case here, $z_{0}$ was of the order of $40 \mathrm{~km}$. This particular normalization of the phase has been used on previous occasions [Wait and Walters, 1963].

Following the usage in previous papers [Wait and Walters, 1963], the quantity $\omega_{r}=\omega_{0}^{2}(0) / \nu(0)$ is specified. In particular, $\omega / \omega_{r}=1 / 2$ at $15 \mathrm{kc} / \mathrm{s}$, or $\omega_{r}=$ $6 \pi \times 10^{4} \mathrm{sec}^{-1}$. The "effective" conductivity $\sigma_{e}$ of the medium at this level $z=0$, is then given by $\sigma_{e}=$ $\epsilon_{0} \omega_{r} \sim 1.7 \times 10^{-6}$ mhos/meter. With exponential-type profiles the fixing of the parameter of $\omega_{r}$ is not an essential restriction. It is a simple matter to shift the reference level from $z=0$ to any other value if desired.

The parameters of the problem are thus $\lambda, C, b, a$, and $\omega_{T} / \nu_{0}$. In order to display the relative influence of these quantities, it is desirable to plot the amplitude and phase of $R$ as a function of $\omega_{T} / \nu_{0}$ from -3 to +3 for a range of values of $\lambda, C, b$, and $a$. It should be noted that $\lambda$ is in $\mathrm{km}, C$ is dimensionless, while $b$ and $a$ have dimensions of $\mathrm{km}^{-1}$. Consequently, the scale length in the present problem is the kilometer. By changing this scale, the results may also have significance at higher frequencies.

In figures $2 \mathrm{a}$ and $2 \mathrm{~b}$ the magnitude of the reflection coefficient $|R|$ and the phase of $R$ are plotted as a function of $\omega_{T} / \nu_{0}$. Negative values of the abscissa correspond to propagation from west to east along the magnetic equator. The cosine angle of incidence is fixed at 0.1. Thus, the angle is highly oblique, being only $5.7^{\circ}$ from grazing. For long-distance propagation of VLF radio waves, such highly oblique conditions prevail. ${ }^{3}$ For the curves in figures $2 \mathrm{a}$ and $2 \mathrm{~b}$, the collision profile is chosen so that the collision parameter $a=0.15 \mathrm{~km}^{-1}$ and the wavelength $\lambda=15$ $\mathrm{km}$ correspond to a frequency of $20 \mathrm{kc} / \mathrm{s}$. For these curves, the electron density parameter $b$ takes the values $0.1,0.2$, and $0.5 \mathrm{~km}^{-1}$. It is evident that for $\omega_{T} / \nu_{0} \cong 0$, the steep gradient of electron density is associated with maximum amplitudes of reflection. However, when $\omega_{T} / \nu_{0}$ is finite, this may no longer be the case. In fact, the asymmetry of the curves about $\omega_{T} / \nu_{0}=0$ is a measure of nonreciprocity in the reflection process. As indicated, the reflection coefficient for propagation from west to east is greater than for propagation from east to west. There is also some nonreciprocity in the phase curves but it is not great.

In figures $3 \mathrm{a}$ and $3 \mathrm{~b}$, a set of curves show the influence of varying the collision frequency parameter while keeping the electron density parameter fixed at $b=0.15 \mathrm{~km}^{-1}$. For these curves, as before, $C=0.1$ and $\lambda=15 \mathrm{~km}$. It is evident that the steeper gradient of the collision frequency corresponds to larger reflection coefficients.

In figures $4 \mathrm{a}$ and $4 \mathrm{~b},|R|$ and the phase of $R$ are shown as a function of $\omega_{T} / \nu_{0}$ for various frequencies in the range from 6 to $60 \mathrm{kc} / \mathrm{s}$. For these curves, $C=0.1, b=0.15$, and $a=0.15$. The tendency is for the reflection coefficient to be diminished at the higher frequencies. In this case, the medium is acting like a good absorber rather than a reflector.

${ }^{3}$ In fact, the attenuation of the dominant mode in the earth-ionosphere waveguide at VLF is approximately proportional to $1-|R|$ for highly oblique incidence [Wait, 1962]. 

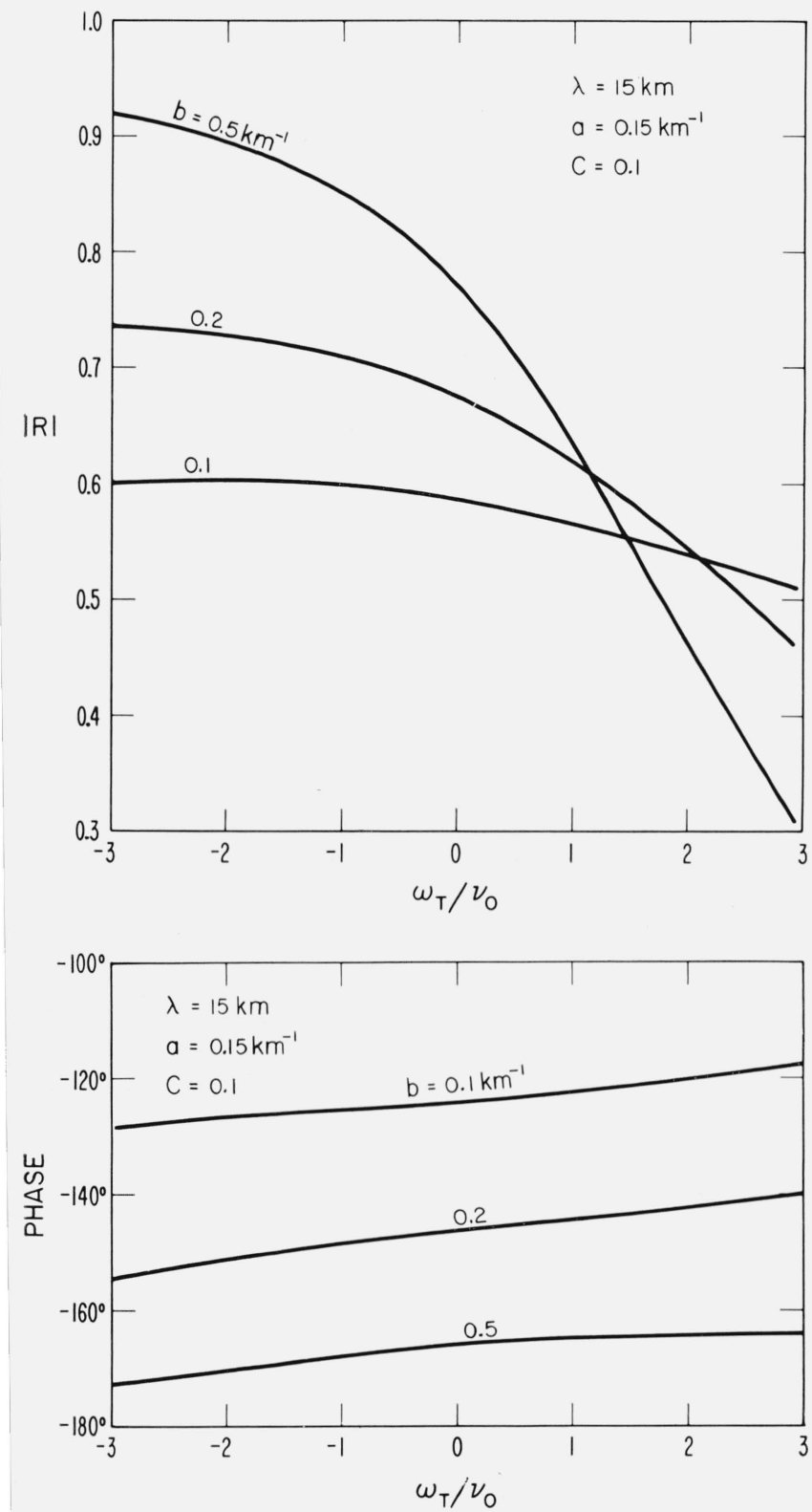

Figures 2a and 2b. Reflection coefficient curves illustrating the dependence on electron density profile.

In figures $5 \mathrm{a}$ and $5 \mathrm{~b},|R|$ and the phase of $R$ are plotted for different values of the angle of incidence. For these curves, $\lambda=15 \mathrm{~km}, b=0.15 \mathrm{~km}^{-1}$, and $a=0.15 \mathrm{~km}^{-1}$. In general, it may be seen that the reflection coefficient is diminished for the steeper angle of incidence. It is rather interesting to note that the asymmetry (or nonreciprocity) in the phase curves is more pronounced at the steeper angles.

Finally, in figures $6 \mathrm{a}$ and $6 \mathrm{~b},|R|$ and the phase of $R$ are plotted for various values of the collision frequency parameter a for $\lambda=15 \mathrm{~km}$ and $C=0.1$. These curves differ from figures $3 \mathrm{a}$ and $3 \mathrm{~b}$ in that here the parameter $\beta=b+a$ is fixed rather than just $a$. In other words, the profile of $N / \nu$ as a function of $z$ is
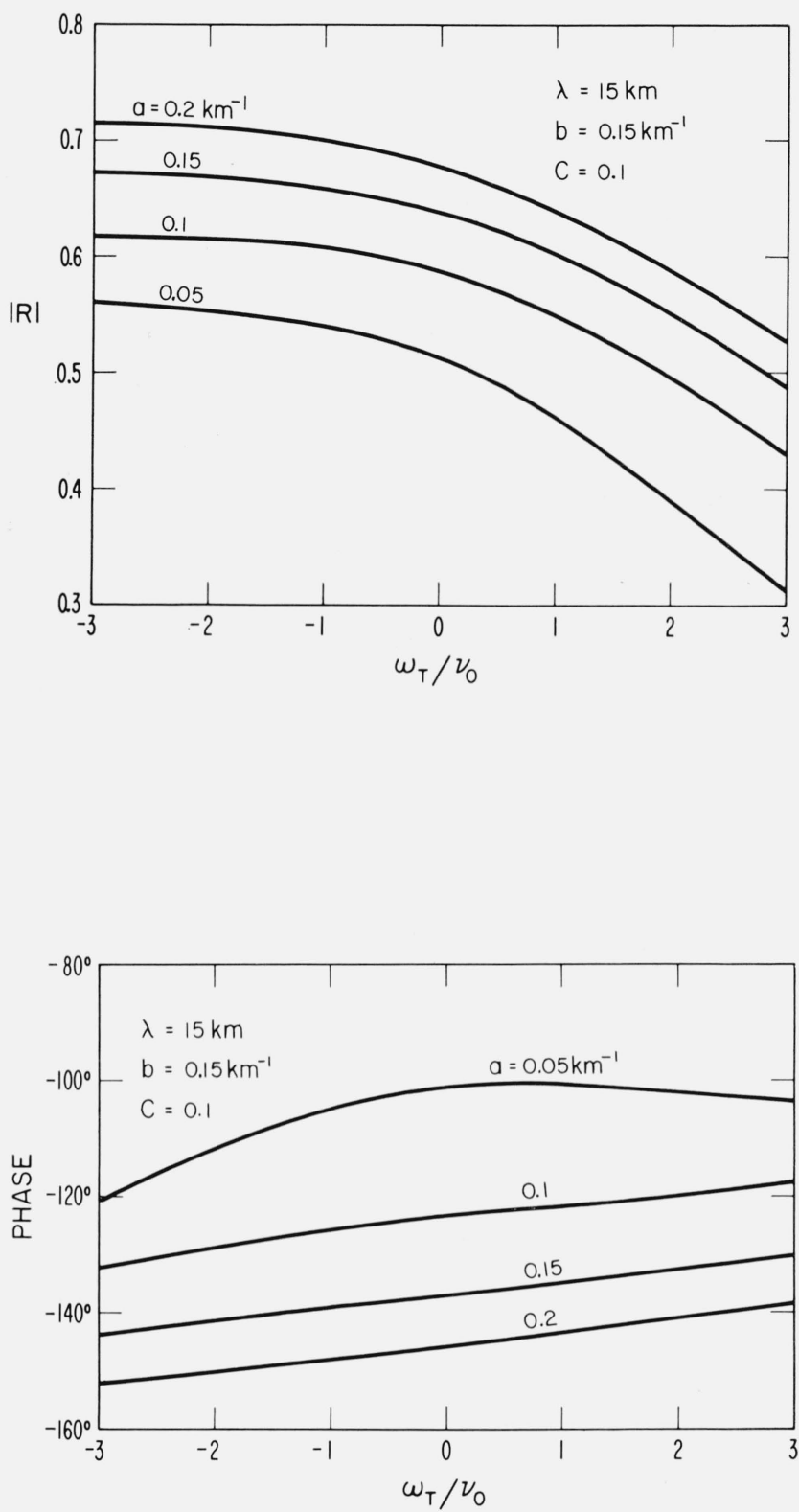

FIgures 3a and 3b. Reflection coefficient curves illustrating the dependence on collision frequency profile.

fixed while the gradient of $\nu$ is changed. In the isotropic case, where $\omega_{T}=0$, it is interesting to note that $R$ is determined only by the gradient of $N / \nu$. However, for a finite gyrofrequency, the situation is changed significantly. In general, the nonreciprocity is accentuated when $\alpha$ is diminished. For example, if $\nu$ were assumed to be a constant, the dependence of the gyrofrequency is much greater than for a collision frequency which varies with height. In much of the earlier work [e.g., Budden, 1955] on full wave solutions in ionospheric radio waves, it is often assumed that $\nu$ can be regarded as a constant. Clearly, such an assumption may lead to very misleading results. 

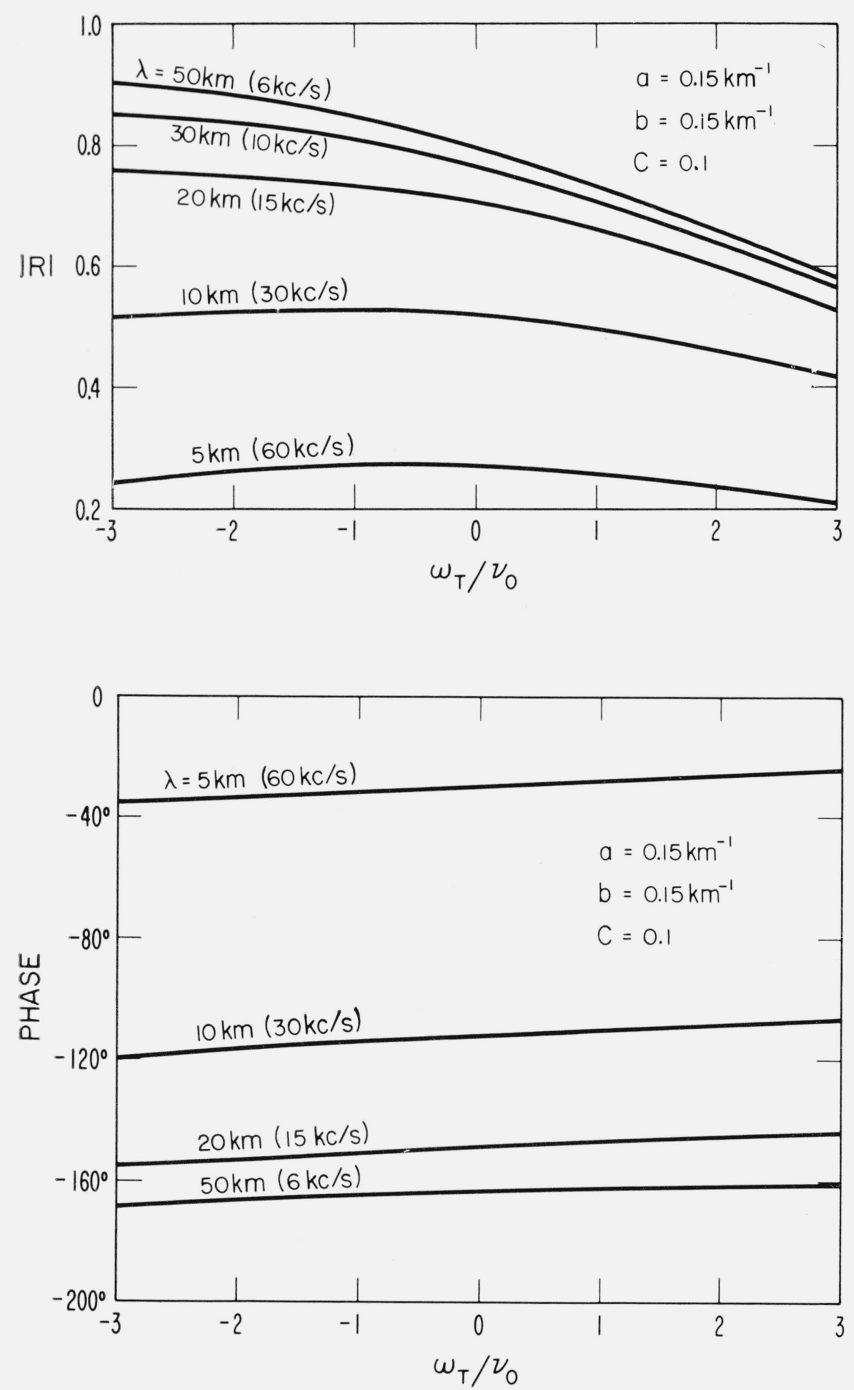

Figures 4a and 4b. Reflection coefficient curves illustrating the dependence on wavelength.

\section{Discussion and Concluding Remarks}

The numerical results given here should provide some insight into the nature of reflection from an inhomogeneous ionized medium. The nature of the dependence on $N, \nu$, and $\omega_{T}$ is quite complicated. Nevertheless, it appears that the sharper gradients of electron density are usually associated with higher reflection coefficients. The dependence on the collision frequency profile is not so clear-cut.

In nearly every case it may be seen that the presence of the transverse magnetic field is to cause the reflection coefficient $|R|$ to be nonreciprocal. Furthermore, for a wide range of the parameters, $|R|$ is greater for west-to-east propagation than for eastto-west propagation. This is in accord with experimental data of Round et al. [1925] who observed that, for propagation over distances of the order of
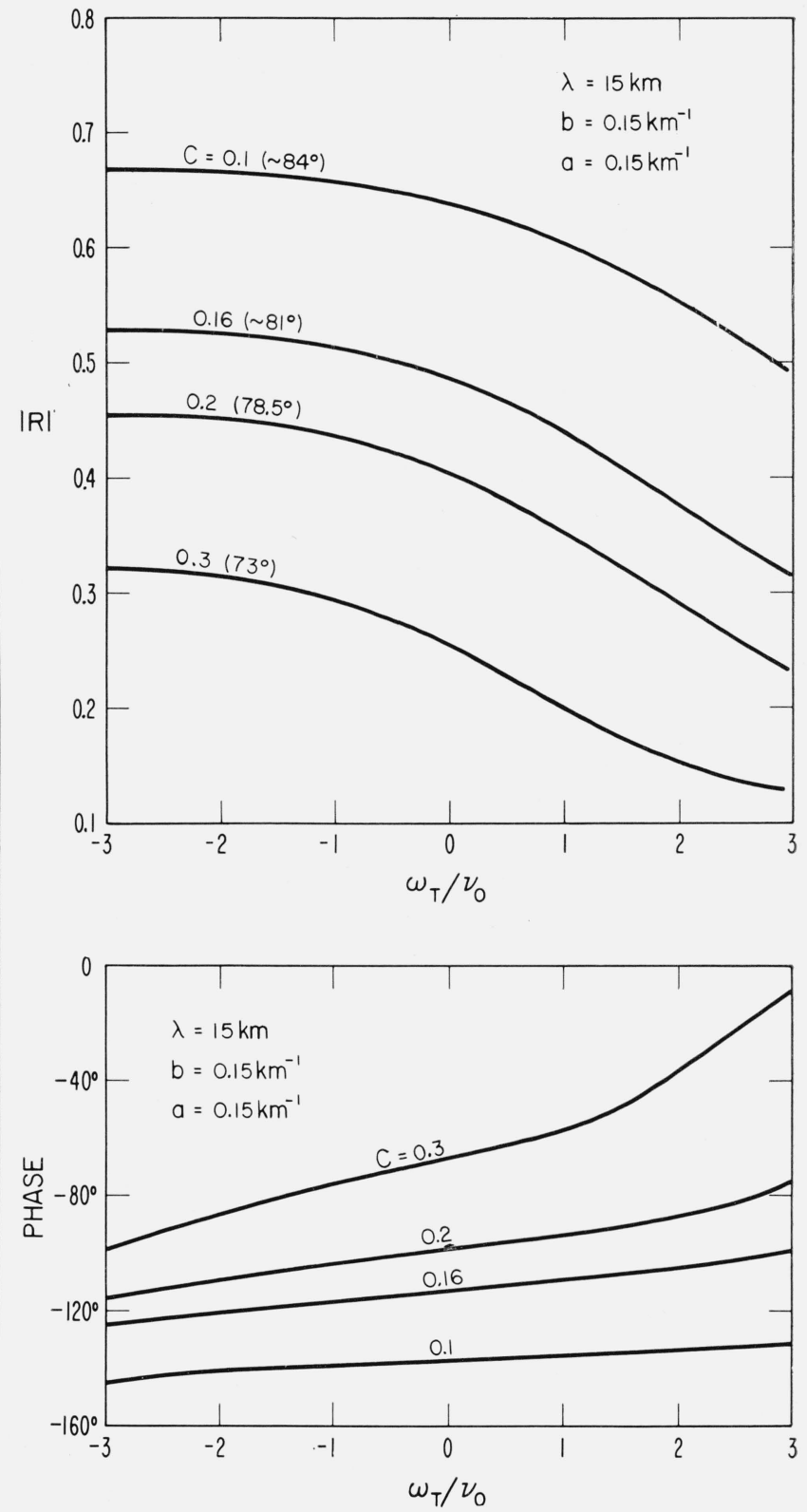

Figures 5a and 5b. Reflection coefficient curves illustrating the dependence on angle of incidence.

$6000 \mathrm{~km}$, signals from VLF transmitters to the west are received more strongly than from those to the east. This observation has also been confirmed by Crombie [1958] in a series of field strength measurements in New Zealand and by Taylor [1960], who analyzed the waveforms of atmospherics. For some inexplicable reason, Budden [1955] deduces from a full wave solution that the directional dependence is just opposite to this. Although his model is not the same as the one considered here, it is difficult for this writer to accept the validity of his results in this regard. However, it is possible that, because of the complexity of the various phenomena, a reversed trend may emerge for certain special conditions, par- 

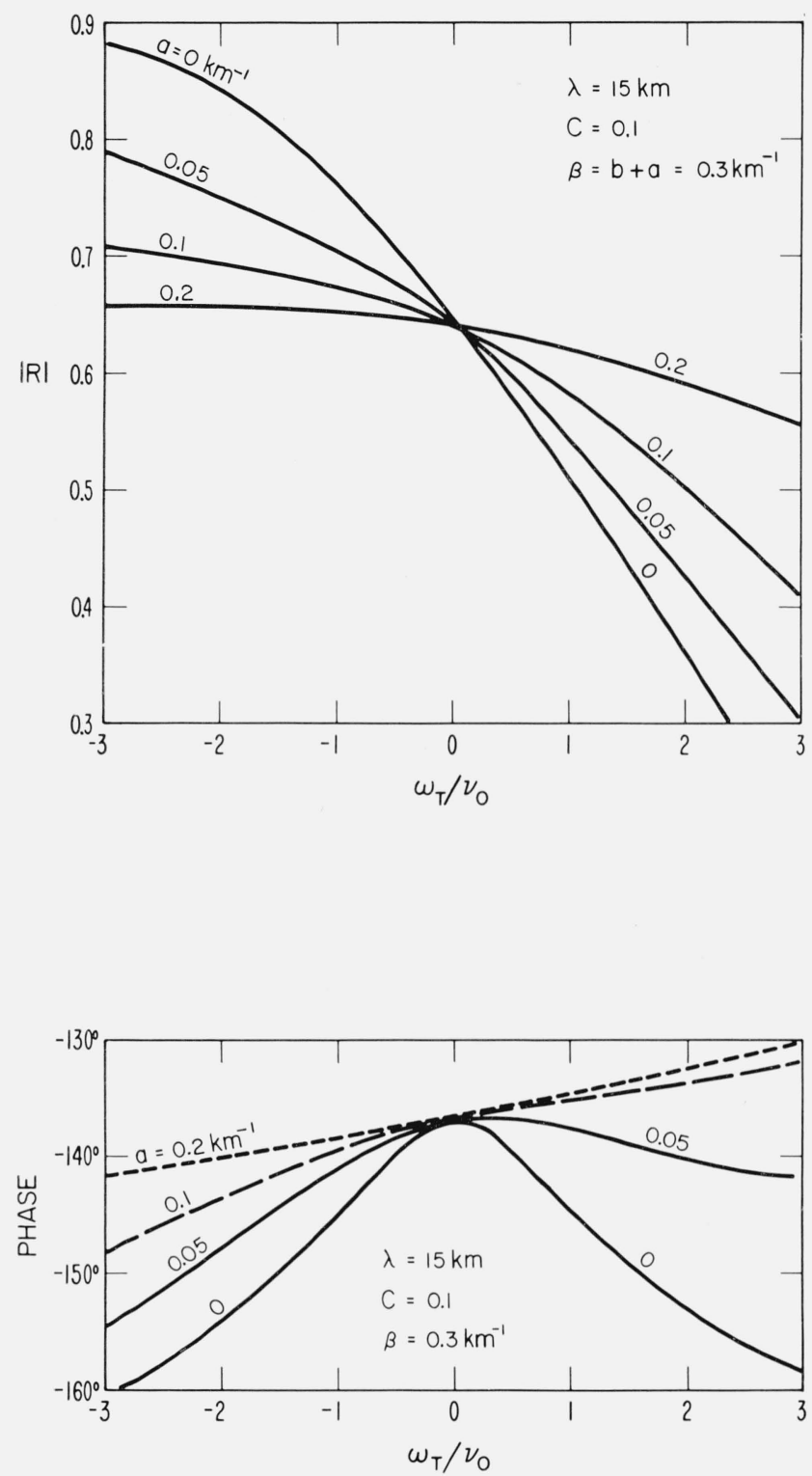

Figures $6 \mathrm{a}$ and $6 \mathrm{~b}$. Reflection coefficient curves illustrating the dependence on collision frequency profile when the $\mathrm{N} / \nu$ profile is fixed. ticularly for the nighttime ionosphere [Rhoads et al., 1963]. It is also worth mentioning that Budden [1955] has some qualms concerning the accuracy of his numerical data at small values of $C$.

\section{References}

Barber, N. F., and D. D. Crombie (1959), VLF reflections from the ionosphere in the presence of a transverse magnetic field, J. Atmos. and Terrest. Phys. 16, 37.

Belrose, J. S. (1963), Present knowledge of the lowest ionosphere, a chapter in Radio Wave Propagation (ed. by W. T. Blackband) (Pergamon Press, Oxford).

Budden, K. G. (May 1955), The solution of the differential equations governing the reflexion of long radio waves from the ionosphere II, Phil. Trans. Roy. Soc. (London), Series A, 248, 45-72.

Budden, K. G. (1961), Radio Waves in the Ionosphere (Cambridge University Press).

Budden, K. G. (1962), The Waveguide Mode Theory of Wave Propagation (Prentice-Hall, Englewood Cliffs, N.J.).

Crombie, D. D. (1958), Differences between east-west and west-east propagation of VLF signals over long distances, J. Atmos. and Terrest. Phys. 12, 110-117.

Johler, J. R. (Jan. 1961), Magneto-ionic propagation phenomena in low- and very-low-radiofrequency waves reflected by the ionosphere, J. Res. NBS 65D (Radio Prop.), 53-61.

Johler, J. R. (1963), Radio wave reflections at a continuously stratified plasma with collisions proportional to energy and arbitrary magnetic induction, Proc. International Conference on the Ionosphere, 436-445 (Chapman \& Hall Ltd., London).

Rhoads, F. J., W. E. Garner, and J. E. Rogerson (1963), Some experimental evidence of direction effects on VLF propagation (private communication).

Round, H. J. T., T. L. Eckersley, K. Tremellen, and F. C. Lunnon (1925), Report on measurements made on signal strength at great distances during 1922 and 1923 by an expedition sent to Australia, J.I.E.E. 63, 933-1011.

Schelkunoff, S. A. (1943), Electromagnetic Waves (Van Nostrand, New York).

Taylor, W. L. (July 1960), VLF attentuation for east-west and west-east daytime propagation using atmospherics, J. Geophys. Res. 65, No. 7, 1933-1938.

Wait, J. R. (1962), Electromagnetic Waves in Stratified Media (Pergamon Press, Oxford, and Macmillan, New York).

Wait, J. R., and L. C. Walters (1963), Reflection of VLF radio waves from an inhomogeneous ionosphere, Parts I, II, III, J. Res. NBS 67D (Radio Prop.), Nos. 3, 5, and 6.

Watson, G. N. (1919), The transmission of electric waves round the earth, Proc. Roy. Soc. 95, 546.

\section{Additional Reference}

Galejs, J. (Nov. 1961), ELF waves in the presence of exponential profiles, IRE Trans. Ant. Prop. AP-9, No. 6, 554562 .

(Paper 68D1-321) 